Годишњак Филозофског факултета у Новом Саду, Књига XLVI-3 (2021)

Annual Review of the Faculty of Philosophy, Novi Sad, Volume XLVI-3 (2021)

Vanja Manić-Matić*

Faculté de Philosophie et Lettres

Université de Novi Sad
UDK : 811.133.1:37

578.834"202"COVID-19

DOI: $10.19090 /$ gff.2021.3.175-190

pregledni rad

\title{
L'ENSEIGNEMENT DU FRANÇAIS LANGUE ÉTRANGÈRE DANS UN CONTEXTE DE PANDÉMIE : ASPECTS TECHNOLOGIQUES ET CULTURELS ${ }^{* *}$
}

Depuis l'apparition du virus COVID-19 et de la crise sanitaire globale, nous vivons dans une nouvelle époque qui nous a imposé de nouvelles habitudes et de nouveaux comportements. La pandémie s'est reflétée dans tous les domaines de la vie, sur le plan de l'enseignement/apprentissage du français langue étrangère (FLE), aussi bien que sur le plan culturel. Les interactions entre les humains ont considérablement changé la manière de penser et de réagir. D'une part, dans l'enseignement/apprentissage du FLE en contexte universitaire aussi, la méthodologie et l'approche pédagogique ont dû être adaptées et sont, par conséquent, devenues virtuelles. D'autre part, les échanges culturels ont trouvé un nouveau biais pour se manifester et pour se développer. L'objectif de ce travail est d'attirer l'attention sur les importants changements liés à la pandémie et enfin développer les consciences autour de ces nouveaux enjeux en vue d'une amélioration et d'un approfondissement de l'enseignement/apprentissage du FLE. En se basant sur notre propre expérience et sur la méthodologie descriptive, nous en donnerons les résultats les plus importants de la recherche : des changements méthodologiques de l'enseignement/apprentissage du FLE en contexte universitaire, ainsi que des changements interculturels où les frontières s'effacent en pandémie.

Mots clés : français langue étrangère (FLE), enseignement/apprentissage, pandémie, technologie, culture.

\section{INTRODUCTION}

Aujourd'hui nous sommes tous témoins de l'ouverture historique d'une nouvelle page dans la civilisation mondiale. Nous pouvons dire que nous avons eu une vie avant et après le coronavirus. Le virus Covid-19 s'est annoncé en Serbie au mois de mars 2020 (le 6 mars) et le 11 mars 2020 la pandémie a été

\footnotetext{
*vanja.manic.matic@ff.uns.ac.rs

* Cet article est rédigé dans le cadre du projet scientifique (1001-13-01) financé partiellement par l'Agence universitaire de la francophonie et l'Ambassade de France en Serbie.
} 
proclamée. Depuis, elle a sillonné le chemin d'une nouvelle ère de pensée et d'interaction, de nouvelles habitudes et de nouveaux comportements. $\mathrm{Vu}$ qu'elle s'est reflétée dans tous les domaines de la vie, elle n'a pas non plus épargné le domaine de l'enseignement/apprentissage des langues étrangères en contexte universitaire. Les professeurs et les apprenants de FLE se sont retrouvés dans une nouvelle situation où ils ont dû s'adapter à une nouvelle forme de classe qui sous-entend les cours à distance. Ainsi donc, l'enseignement du FLE est devenu non seulement un lieu d'interaction virtuel mais aussi un lieu d'activité sociale particulière.

Le travail ci-présent a notamment pour objectif de donner un aperçu des aspects technologiques et culturels qui se sont répercutés dans la didactique du FLE lors de la pandémie. La question que l'on pose ici est la suivante : est-ce qu'il est possible d'enseigner de la même manière qu'avant la période de la pandémie, et est-ce qu'on peut transmettre, varier les différents points de vue d'une culture étrangère aux apprenants du FLE ? On s'attend que chaque enseignant sache faire face à de nouvelles situations comme si cela était inné et naturel pour lui, mais est-ce toujours le cas? Nous ne nous demandons jamais combien d'efforts physiques et psychologiques sont nécessaires à la bonne réalisation d'un cours, surtout aujourd'hui, en pleine pandémie. C'est pour cela que nous allons d'abord présenter les nouveaux défis technologiques dans la réalisation des cours, ensuite nous allons voir comment de nouvelles manifestations socioculturelles ont trouvé leur chemin pour se développer en général et comment on pourrait bien les exploiter dans un cours de langue. De même, tout au long du travail, nous allons nous baser sur notre expérience d'enseignante en contexte universitaire, pour pouvoir enfin en ressortir des conclusions générales qui pourront aider et améliorer le futur enseignement/apprentissage du FLE.

\section{ASPECTS TECHNOLOGIQUES - UNE NOUVELLE ORGANISATION DES COURS}

Dès la proclamation de la nouvelle situation sanitaire et du confinement on a dû s'adapter aux nouvelles conditions de vie dans tous les domaines. L'éducation non plus n'était pas épargnée et la classe traditionnelle a rapidement été remplacée par la classe virtuelle. L'enseignement en ligne a connu un essor rapide suite à l'annonce du confinement. Il était très important de prendre toutes les précautions contre la transmission du virus. Dans notre 
cas universitaire l'enseignement en présentiel a dû être remplacé par l'une des variantes de l'enseignement en ligne. Cela sous-entendait une nouvelle gestion de la classe, c'est-à-dire une nouvelle approche méthodologique. Nous savons que depuis la publication du CECRL (Cadre européen commun de référence pour les langues) le manuel en tant que support pédagogique n'était pas suffisant, qu'on devait toujours y ajouter des documents authentiques. D'après le CECRL on doit « traiter les compétences langagières dans un cours de langue [...] par le contact direct avec des locuteurs natifs et des textes authentiques » (CECRL, 2000 : 113). Cependant, maintenant dans les nouvelles conditions, on en est devenu beaucoup plus conscient. L'adaptation aux cours virtuels pendant le confinement était un défi aussi bien pour les enseignants que pour les apprenants.

Par ailleurs, il faudrait aussi tenir compte de plusieurs facteurs qui avaient un grand rôle sur le développement et la réalisation des cours en ligne : l'état physique, l'état psychique, l'interaction: enseignants/apprenants, les moyens techniques, l'organisation et l'adaptation de l'enseignement du FLE en ligne et le choix de la plateforme.

En ce qui concerne le premier facteur, "l'état physique», il faudrait mentionner que la plupart des étudiants ont dû se déplacer, rentrer chez eux, et certains d'entre eux ont été contaminés par le virus, c'est-à-dire dans l'impossibilité de suivre les cours. Ceux qui sont rentrés, se sont retirés chez eux en vitesse, alors majoritairement ils n'avaient pas apporté leurs manuels, il était donc nécessaire de bien faire le choix des documents pédagogiques et de bien les envoyer à tout le monde. En parlant du deuxième facteur, de « l'état psychique », nous avons bien senti une baisse d'énergie, de moral, un certain stress, une tension et parfois une peur de l'inconnu. Alors, le rôle de l'enseignant était de bien encourager tout d'abord soi-même, ensuite les apprenants et de les motiver à s'intégrer aux cours en ligne. De même, l'interaction enseignants/apprenants a aussi bien changé. De la part de l'enseignant, on avait parfois l'impression d'être dans une classe noire, parce que certains étudiants ne voulaient pas non plus être visibles à l'écran. Pourtant, pour un suivi dans l'apprentissage d'une langue étrangère, on devait avoir un contact virtuel direct, car il s'agissait bien de la communication non 
verbale qui est à nos yeux ${ }^{1}$ très importante dans l'apprentissage. Au sujet des moyens techniques, le manque de bons outils comme l'ordinateur, la caméra, le son, etc. posait aussi d'importants problèmes. À cela il fallait encore ajouter le fait d'une mauvaise connexion qui empêchait un bon suivi du cours, non seulement de la part des apprenants, mais aussi de la part de l'enseignant, ce qui parfois était encore pire, car c'était surtout lui qui orchestrait toute la classe.

Le dernier enjeu, qui aurait pu aussi être le premier, était la question : comment organiser et adapter l'enseignement du FLE en ligne : via le courriel, les plateformes Moodle ou Zoom, etc. ? Nous allons dédier un peu plus d'attention à ce facteur dans les paragraphes qui suivent.

\subsection{Le courriel}

Tout d'abord, il faudrait dire que, dans le contexte universitaire, nous nous sommes décidés pour le moyen de communication le plus simple et celui auquel les étudiants étaient déjà sensibilisés auparavant, complémentaire aux cours tenus en présentiel, c'est-à-dire pour le courriel ou e-mail. La différence, entre son utilisation d'avant et celle en temps de pandémie, est que dans ce deuxième cas de figure il fallait bien structurer et choisir les supports pédagogiques complémentaires au manuel et éviter en même temps l'encombrement de documents. De même, les supports pédagogiques devaient être bien préparés cette fois-ci dans un format digitalisé et présentés sous forme de petits textes authentiques et variés, enregistrements audio et/ou visuels, d'un roman feuilleton, de chansons, d'extraits vidéo, d'exercices interactifs en ligne TV5 monde (dictée ou grammaire) etc. Il était très important de ne pas charger les étudiants avec une multitude de choix, ce qui était difficile et un vrai défi. Or, un bon enseignant devrait montrer cette subtilité et ne pas prendre une place de supérieur vis-à-vis de ses apprenants, mais bien les mener et guider vers une nouvelle approche de l'apprentissage. Donc, il était nécessaire de prudemment choisir les supports pédagogiques, de bien les structurer et enfin de les envoyer. Ici aussi il faudrait tenir compte du

${ }^{1}$ L'auteure de cet article a déjà abordé ce sujet dans l'article « Le (non) verbal dans l'enseignement du FLE - enjeux, défis et réalités en contexte universitaire», paru in : Godišnjak Filozofskog fakulteta u Novom Sadu, XLV-4 (2020), 261-271. 
fait que même la liste des étudiants avec leurs adresses électroniques n'était pas toujours la bonne et ajustée, soit à cause de leur absence, soit à cause de leur manque de supports techniques. Alors, il a fallu attendre que les étudiants s'annoncent tout seuls directement, ou par le biais des autres étudiants, leurs collègues, pour qu'on puisse vraiment réaliser le travail en cours dans les nouvelles conditions.

\subsection{La plateforme Moodle}

La plateforme Moodle est l'une des plus répandues au monde et compte plus de 32 millions d'utilisateurs. Elle a été adoptée par de nombreuses institutions dans tous les domaines surtout grâce à son utilisation facile et intuitive. Depuis quelques années, ce programme de cours à distance est complémentaire et soutenu par La Faculté de Philosophie et Lettres à Novi Sad aussi. Il faudrait mentionner que c'était un grand avantage pendant le confinement pour les enseignants qui avaient déjà intégré Moodle à leurs cours habituels avant la pandémie, parce qu'ils ont seulement continué à l'utiliser. Pour d'autres enseignants son utilisation au début était un défi, ensuite elle est devenue une habitude et une bonne pratique qu'on continue à exercer toujours.

La plateforme Moodle est favorable pour plusieurs raisons : elle est gratuite, facile et logique à utiliser, permet de bien viser la tâche (conférences enregistrées, devoirs, enquêtes, examens partiels, tests, etc.), elle offre la possibilité de déposer et créer des ressources pédagogiques (fichier, étiquette, dossier, livre, page, URL, vidéo, son), elle stimule le travail collaboratif, elle offre une modification ou une adaptation facile des cours. Pour y accéder il faut d'abord administrer les cours : paramétrer les cours, inscrire des étudiants, sauvegarder les cours, gérer le profil. De même, grâce à cette plateforme on peut aussi personnaliser les cours, c'est-à-dire organiser et nommer les sections, gérer les blocs ou utiliser le forum des nouvelles. Cependant, elle a aussi un inconvénient: dans un sens elle reste passive par rapport à l'interaction réelle et directe en ligne entre l'enseignant et l'apprenant. C'est pour cela que dans notre cas Moodle était complémentaire et seulement une des variantes de l'enseignement en ligne avec le courriel et la plateforme Zoom. 


\subsection{La plateforme Zoom}

Nous avons utilisé cette plateforme pour plusieurs raisons : inscription gratuite, simple installation (téléchargement de l'application gratuit), utilisation simple, durée d'une session gratuite presque comme celle d'un cours (40 minutes), joignable via une webcam ou un téléphone, visioconférences de groupes - 100 personnes à la fois (créer une nouvelle réunion, en planifier une, rejoindre une réunion, créer des réunions récurrentes), possibilité de répartir un groupe en plusieurs - travail en minisections et supervision.

Pour ce qui est des paramètres, nous sommes d'avis que grâce aux différents onglets nous avons l'impression de travailler presque dans une vraie classe de FLE, car ils disposent de fonctions diverses comme: "partager l'écran» (partage/suspension d'écran - remplacement du tableau), « converser/chat» (enregistrement du chat/correction simultanée en ligne), "réactions" (réagir avec un émoticone/exprimer son émotion), «muet» (microphone activé/désactivé - possibilité de donner la parole à quelqu'un), « enregistrer » (vidéo, audio, texte du chat), " démarrer/arrêter la vidéo », « inviter »/« participants » (ajout facile des contacts), " quitter la réunion », " quitter le mode plein écran », " affichage intervenant », arrière-plans virtuels (pour cacher le désordre), retouche de l'apparence.

Dans le cas de nos cours à distance, la plateforme Zoom était une bonne complice pour l'enseignement en ligne - un outil supplémentaire et accessible facilement depuis tous les dispositifs mobiles (ordinateur, téléphones portables, tablettes). Les étudiants qui ne pouvaient pas rejoindre un cours sur une plateforme, avaient le choix de le faire autrement. De même, cette plateforme était un lien de solidarité, de soutien et d'encouragement. Elle offrait une possibilité de rencontres et d'échanges, de classe virtuelle et de réunions, non seulement entre un enseignant et des apprenants, mais aussi entre des enseignants eux-mêmes. Et enfin, c'était un lien de partage d'idées à travers les nombreux webinaires, c'est-à-dire visioconférences (séminaires en ligne), organisés pour la promotion de l'enseignement/apprentissage du FLE de la part de nombreuses institutions francophones et non francophones (Institut français, Université de Novi Sad, Bibliothèques universitaires, etc.). 


\subsection{Des avantages et des inconvénients de l'enseignement/apprentissage en ligne}

Après presque un an d'enseignement en ligne nous pouvons dire que ce type de cours à distance a bien des avantages ainsi que des inconvénients. Il faut aussi tenir du compte du fait qu'on peut aborder cette problématique de deux côtés, c'est-à-dire de la part de l'enseignement et de l'apprentissage.

Pour ce qui est de l'enseignement à distance, la flexibilité, l'accès de n'importe quel endroit ainsi que l'efficacité font partie de ses avantages. Cependant, ce qui s'est avéré être un inconvénient c'est l'interaction directe qu'il est beaucoup plus difficile à susciter, ainsi que la baisse de la motivation chez des apprenants. De même, les cours à distance sont beaucoup plus exigeants car ils sont plus intensifs, alors il est nécessaire de faire des pauses pour éviter la fatigue et la satiété de l'enseignement. À cela nous pouvons aussi ajouter les préparatifs pour les cours qui sont également beaucoup plus complexes, puisque dans la classe traditionnelle chaque enseignant pouvait plus spontanément faire une petite pause pour reprendre son souffle, ce qui n'était pas le cas pour la classe virtuelle où chaque pause apparaissait plutôt comme une méconnaissance, ou encore comme un manque de crédibilité. Ce qu'il faudrait aussi ajouter ce sont les évaluations et les corrections des copies des apprenants, qu'il était difficile de contrôler. Nous nous sommes aperçue que le fait de « copier et coller » d'une majorité des apprenants était très abusé et que les vrais résultats n'arrivaient que lors de leurs examens, tenus à la faculté en présentiel, et non pas à distance.

Alors, c'est ainsi qu'on passe à l'autre point de vue, à l'apprentissage, qui est dans un sens devenu effet à double tranchant. En copiant, les apprenants avaient une fausse impression sur leurs savoirs, et ils étaient assez déçus en apprenant où ils en étaient après avoir eu les notes de leurs examens. Un autre problème dans ce domaine était aussi leur engagement, qui de la part de certains était assez faible, disons inerte. En s'investissant peu physiquement et mentalement en cours à distance, il y avait moins d'interactions et par conséquent, moins d'apprentissage. Ici nous avons fait face à une problématique: le phénomène $\mathrm{du}$ savoir-faire, indispensable pour l'apprentissage, et la question de comment « apprendre à apprendre » dans les nouvelles circonstances. Dans un sens, les apprenants ont commencé à se perdre dans leur apprentissage, tandis qu'il fallait qu'ils deviennent presque autodidactes, bien sûr avec un certain guidage de leur enseignant. Ce 
phénomène assez complexe, nous allons le laisser pour une étude ultérieure. Quoi qu'il en soit, les apprenants aussi bien que les enseignants devaient s'organiser et s'engager davantage dans ce type de cours qui imposaient une certaine littératie numérique et qui n'était pas du tout un impératif dans une classe traditionnelle.

\section{ASPECTS CULTURELS - UNE NOUVELLE MANIÈRE DE PENSER}

Depuis l'apparition du coronavirus la vie a changé globalement. Les nouvelles circonstances dues à la crise sanitaire globale ont imposé nécessairement de nouveaux comportements. Les interactions entre les humains ont beaucoup évolué, de même que la manière de penser et de réagir. D'après Geneviève Zarate «la description valorisante de la culture étrangère peut également contribuer à la valorisation de la culture nationale locale » (Zarate, 2016 : 23). Cependant, il semble que dans la pandémie et pour la première fois enseigner une culture devient un phénomène unique dans un sens, car les cultures dans ce cas particulier ont commencé à se ressembler et donc les frontières entre elles semblaient s'effacer spontanément : « [...] les représentations sociales ne sont pas une collection de subjectivités distinctes, mais au contraire le produit d'un travail social collectif, à travers lequel les agents sociaux construisent leurs modes de connaissances de la réalité » (Zarate, 2016 : 29). Dans notre cas, la pandémie comme crise sanitaire globale est apparue en effet comme un atout, car toutes les personnes confinées ont commencé à s'exprimer, à se manifester d'une même façon et à avoir malheureusement une même vision du monde. Alors cette connaissance de l'Autre en tant que phénomène de l'interculturalité est en effet devenue transparente et unique pour toutes les cultures, qui de leur part avaient seulement quelques comportements plus ou moins accentués par rapport à une autre culture étrangère. Dans ce sens-là, la perception de la nouvelle culture étrangère, française notamment, était rassurante pour l'apprentissage du FLE dans notre contexte, car les serbophones étaient pour la première fois dans une telle situation ainsi que le monde entier, ce qui durant leur histoire n'était jamais le cas. À cause de leur histoire dure et complexe, ils se sont toujours sentis culturellement inférieurs par rapport à certaines grandes cultures de l'ouest, ce qui était complètement différent cette fois-ci et par conséquent favorable pour la motivation de l'enseignement et l'apprentissage 
du FLE : «[...] dans notre passé la langue française a été depuis longtemps considérée comme la langue internationale et diplomatique, et parfois au détriment de l'acception positive de la culture maternelle » (Manić-Matić, 2017 : 20). « La représentation de la civilisation étrangère dans l'enseignement du FLE sur notre territoire se souciait très peu du fait qu'elle pourrait développer ou repousser la conscience des qualités et des valeurs de la civilisation maternelle chez les apprenants» (Polovina, 1980: 139)2. Par ailleurs, depuis les années 80 cette situation a changé, notamment depuis la période de la décolonisation, et surtout depuis la fin des années 90, « avec les méthodes récentes, basées sur l'approche communicative, qui la favorisent à travers l'utilisation de documents authentiques " (Manić-Matić, 2017: 21). Même si aujourd'hui la francophonie est beaucoup plus représentée dans les manuels du FLE, pour certains apprenants serbophones elle reste toujours lointaine et inconnue. Il est important que « deux cultures (ou même plusieurs) se trouvent en contact spatio-temporel très proche, où on aura des transferts de modèles culturels d'un environnement à l'autre » (Durbaba, 2016: 116) ${ }^{3}$. De même, « dans le choix de l'enseignement des cultures étrangères [...] la question de traiter des phénomènes négatifs dans un environnement culturel n'est pas sans importance » (Durbaba, $2016: 80)^{4}$ :

[...] omettre certains éléments de la réalité constitue non seulement sa falsification, mais pourrait conduire à une double frustration : l'apprenant, d'une part, est laissé dans la conviction qu'en dehors de son environnement il existe un autre monde parfait [...]; d'autre part, le risque de choc culturel augmente en cas d'éventuelle confrontation effective avec une culture donnée [...], car ce n'est qu'alors que l'apprenant ferait face à la réalité qui lui avait été soigneusement cachée pendant les cours. (Durbaba, $2016: 80-81)^{5}$.

${ }^{2}$ Citation originale : „Prikazivanje strane civilizacije u našoj nastavi se malo brinulo o tome da li će kod učenika razviti ili potisnuti svest o vrednostima o domaćoj civilizaciji" (Polovina, 1980 : 139).

3 Citation originale: „Ukoliko se dve kulture (ili više njih) nalaze u vremenski i prostorno bliskom kontaktu, neminovno će dolaziti do transfera kulturnih obrazaca iz jedne sredine u drugu [...]" (Durbaba, $2016: 116)$.

${ }^{4}$ Citation originale : , [...] o izboru elemenata inostrane kulture [...] nije bez značaja ni pitanje tretiranja negativnih pojava u jednoj društvenoj sredini [...]" (Durbaba, 2016 : 80).

${ }^{5}$ Citation originale : „[...] izostavljanje određenih elemenata stvarnosti ne samo što predstavlja njeno falsifikovanje, već bi moglo dovesti do dvostruke frustracije : učenik 
C'est la raison pour laquelle il est préférable d'introduire certains phénomènes socioculturels qui ne sont pas nécessairement positifs, mais qui sont d'autre part communs dans leur culture, et de cette façon rassurer et motiver davantage les apprenants dans l'apprentissage du FLE. Passons maintenant à quelques exemples de phénomènes socioculturels qui se sont le plus distingués et qui sont alors devenus uniques pour la culture d'origine et la culture étrangère cible.

\subsection{Les gestes barrières}

Ces gestes se sont les premiers incrustés dès la première vague de l'arrivée du coronavirus. En plus, durant le premier couvre-feu ils étaient même exagérés car les gens avaient peur de l'inconnu et de la crise sanitaire globale. «Se laver les mains très régulièrement; tousser ou éternuer dans son coude ou dans son mouchoir ; utiliser un mouchoir à usage unique et le jeter ; saluer sans se serrer la main, éviter les embrassades ${ }^{6}$, ce ne sont que les conseils primaires dans le monde entier pour se protéger et protéger les autres. De cette façon ils sont favorables, puisque uniques pour tous, à être utilisés et exploités en cours de FLE selon le niveau des apprenants envisagés ${ }^{7}$. Alors à ces gestes on peut toujours ajouter d'autres gestes barrières comme "se désinfecter les mains» ou " porter un masque», qui sont devenus inévitables et tout à fait habituels dans les nouvelles circonstances.

\subsection{La distance physique ou la distance sociale?}

Maintenez-vous une distance physique partout où vous allez ? Faut-il maintenir une distance physique ou sociale? Les questions que l'on se pose tout le temps en pandémie. Il ne faut pas oublier que l'homme est un être social et que dans sa nature il a besoin de communiquer et d'avoir des interactions

se, s jedne strane, ostavlja u uverenju da - izvan njegovog okruženja - postoji neki drugi, savršen svet [...]; s druge strane, povećava se rizik kulturnog šoka u slučajueventualnog stvarnog suočavanja s datom kulturom [...], pošto bi se učenik tek tada suočio s realnošću koja je tokom nastave od njega bila brižljivo skrivana" (Durbaba, 2016 : 80-81).

${ }^{6}$ Il s'agit des consignes figurant sur l'affiche intitulée «Coronavirus, pour se protéger et protéger les autres », réalisée par Santé publique France.

${ }^{7}$ Nous sous-entendons ici les niveaux d'apprentissage prévus par le CECRL. 
avec d'autres êtres et non pas seulement les humains. La nécessité de participer activement à la vie sociale pendant la pandémie a créé cette ambiguïté s'il fallait ou ne fallait pas participer et comment participer à cette vie sociale, quand on est hors de la période du couvre-feu. Il s'agit ici d'un bon sujet qui pourrait toujours être lancé en cours de FLE et qui est propice à un bon débat ou encore une expression écrite critique et bien argumentée.

\subsection{Zoom ou Skype - de nouveaux savoir-vivre}

Les deux plateformes servant de: salle de classe, salle de concert, studio de yoga, ou de gym, table de poker, d'église (des couples qui s'y marient, des familles assistant aux funérailles d'un proche), skypéro (pour sortir et boire devant l'écran). Nous parlons ici de quelques phénomènes sociaux qui pourraient aussi être un sujet pour l'expression orale ou écrite, ou encore lancés pour entamer un bon débat en cours de FLE.

\subsection{De nouvelles applications et émojis}

Pendant le confinement les applications comme Coronavirus (pour tracer et suivre le virus), StopCovid France (pour se protéger et protéger les autres), ou encore des émoticones ou émojis (pour exprimer différentes émotions) ont commencé à apparaître sur des dispositifs mobiles, avec de petites variations, pour rassurer et aider les gens dans la pandémie. Chaque culture les a adoptés à sa façon et étant donné qu'on vit dans un monde digitalisé où on ne connaît pas de barrières, ils sont devenus uniformes pour tous. Eux aussi pouvaient être exploités différemment en cours de FLE, selon le niveau prévu.

\subsection{L'humour, le vrai et l'humour noir}

L'humour, le vrai ou le noir, pendant la pandémie est exprimé sous toutes les formes et sur les différents réseaux sociaux, touchant différents domaines artistiques (peinture, affiches, BD, photo, etc.). Nous allons mentionner quelques exemples et voir quelques traits comiques qui y sont représentés.

L'image qui a beaucoup circulé lors de la pandémie était celle de « La tenue parfaite pour le télétravail - zone visible à la webcam - zone cachée à la webcam ». Dans cet exemple, il s'agit d'une photo où deux jeunes gens 
travaillent de chez eux, mais on peut voir que dans leur «zone visible à la webcam » ils portent des costumes, mais dans la «zone cachée à la webcam » ils ont des pyjamas. En parlant de l'humour et de l'image, on peut travailler toujours sur l'enrichissement du vocabulaire vestimentaire, et donc ils sont favorables dès le niveau débutant d'apprentissage du FLE.

Un autre exemple comique et ironique est le tableau de René Magritte (artiste surréaliste belge), connu par ses trahisons des images et notamment par son tableau « Ceci n'est pas une pipe » (1929) à l'instar duquel est réalisé, et aussi adapté à la nouvelle situation pandémique, le tableau concernant l'enseignement «Ceci n'est pas une école». Sur ce tableau on voit une dame, qui représente une enseignante, devant l'image de trahison incarnée par un ordinateur portable et l'inscription mentionnée. C'est aussi un bon exemple de l'art qui pourrait être exploité en cours de FLE et où on peut lancer le sujet sur l'école d'autrefois et aujourd'hui.

Ensuite, nous allons ici prendre en compte une planche de la bande dessinée "Astérix et Obélix », les représentants stéréotypés de la culture et civilisation françaises. Elle est intitulée «Organisation du déconfinement à la française » et pourrait être adoptée par toutes les cultures confinées. En effet, sur cette image nous pouvons voir une foule de Gaulois qui se précipitent pour faire la fête dès la proclamation du déconfinement. Une image qui pourrait être bien adoptée par les apprenants serbophones car la représentation culturelle de l'Autre est presque identique à leur culture maternelle. Il s'agit d'un bon support pour traiter l'(inter)culturalité.

Pour ce qui est des blagues, nous allons en mentionner deux. Leur exploitation pourrait être différente et dépendrait du niveau prévu. Dans la première, il s'agit d'une image d'un couple obèse confiné dans son appartement, où l'homme, à la fin du confinement, demande à sa femme : «On va sortir comment?», et la femme ne donne pas de réponse mais a seulement un visage qui reflète un point d'interrogation.

L'autre blague ne contient pas d'image mais seulement le texte : « Tu fais quoi cet été ? - Je vais surfer. - Biarritz, Arcachon ? - Non, non, Google, Facebook, YouTube...». Ici il s'agit d'un jeu de mots et de circonstances. De même, c'est aussi un bon exemple de l'emploi polysémique du mot "surfer » que les apprenants du FLE peuvent bien avoir dans un même contexte.

Dans tous ces exemples il faudrait avoir en vue que l'acceptation de l'humour pourrait aussi être relative et va dépendre de chaque individu et de sa propre expérience. C'est pour cela que l'enseignant devrait bien choisir ces 
exemples, selon le niveau, l'âge et les situations vécues dans le groupe où il enseigne, car certaines choses qui sont plaisantes ou drôles pour une personne, ne le sont pas nécessairement pour une autre.

\subsection{La musique}

Nous parlons ici du domaine d'art le plus souvent exploité. Le chant du balcon, les concerts virtuels ou dans des cours entre les immeubles, ce ne sont que quelques manifestations musicales en pandémie. En effet, les artistes du monde entier témoignent de la créativité et de la solidarité. Parfois, ils le font aussi en s'enregistrant et en diffusant leurs vidéos sur différents réseaux sociaux. La musique incite les gens à s'exprimer et ainsi a un impact positif sur l'ensemble des manifestations conscientes et inconscientes de l'être humain. Elle développe une nouvelle esthétique dans sa nouvelle forme, encourage et rassure les gens confinés avec son humour. De même elle représente une diversité culturelle et linguistique et dévoile ainsi tout un nouveau monde.

C'est le cas dans l'exemple de la chanson que nous avons prévue comme support pédagogique authentique pour un niveau A2/B1, prévu par le CECRL. Il s'agit de la jeune chanteuse québécoise de Montréal, Roxane Bruneau et sa chanson "Quarantaine ». Elle a été découverte grâce à Internet et connue pour ses sketchs et chansons partagés sur Facebook, YouTube et Tik-Tok. Ses chansons comptent des millions de vues. La chanson «Quarantaine » est sortie le 26 mars 2020 et elle est utilisable en cours de FLE pour plusieurs raisons : sa durée (1 minute); elle représente un bon exemple de la francophonie confinée; elle est moderne et contemporaine par conséquent attrayante surtout pour le jeune public (YouTube/Tik-Tok) ; pleine d'humour noir ; riche en vocabulaire: lexique de la pandémie, lexique du français de Montréal, lexique du français familier, lexique du contexte culturel du Canada francophone.

Dans cet exemple, le Canada francophone géographiquement loin et inconnu de la part du public serbophone, devient proche grâce à la pandémie, où les frontières s'effacent presque entre toutes les cultures francophones, ainsi vers la nôtre aussi. Cependant, nous pouvons y trouver un vocabulaire qui est réservé et particulier à la culture du Canada francophone où l'Autre est un 
peu différemment accentué. Les paroles de cette chanson, ainsi que le lexique français québécois démontrent bien cette variété culturelle francophone ${ }^{8}$.

\section{CONCLUSION}

L'enseignement du FLE n'a jamais été un si grand défi que dans le contexte de pandémie. Enseigner et penser autrement est devenu un impératif et tous les actants dans l'apprentissage des langues en sont devenus témoins. La classe traditionnelle est devenue désormais virtuelle, et on a dû s'adapter aux nouvelles circonstances subitement. Les aspects que nous venons d'évoquer, aspects technologiques et culturels, sont ceux qui nous ont le plus marquée dans notre contexte universitaire de l'enseignement/apprentissage du FLE. La sensibilisation à l'enseignement en ligne et sa pratique répétitive nous ont menés vers une habitude et une nouvelle approche méthodologique en classe à distance, qui est devenue maintenant quelque chose de normal et de tout à fait ordinaire et naturel. Par ailleurs, en tant qu'enseignants nous avons été témoins qu'avec une pratique plus intensive de l'enseignement en ligne, les apprenants avaient une fausse impression sur leurs savoirs, surtout à cause de leur faible engagement et incompatibilité du système éducatif qui offrait les cours en ligne et la passation des examens en présentiel. De même, nous avons vu que le phénomène de la pandémie nous a apporté encore une nouveauté dans le sens des manifestations socioculturelles. Vu le fait qu'il s'agit d'un fait unique et global, la connaissance de l'Autre dans cette situation est devenue presque transparente, c'est-à-dire que les frontières entre certaines cultures ont tendance à s'effacer, ou sont au moins très faibles, ce qui représente un bon déclencheur pour la motivation dans l'apprentissage du FLE. Somme toute, la nouvelle crise sanitaire nous enseigne qu'il ne faut pas rester inerte, qu'il faudrait toujours s'investir, physiquement et mentalement, sortir de la zone de confort, car c'est ainsi qu'on donne un bon exemple aux apprenants et aux collègues enseignants, et de cette manière on ne cesse pas d'apprendre.

8 Bruneau, R. (26 mars 2020). Quarantaine [vidéo]. Disponible sur : https://www.youtube.com/watch?v=ccAkxNlumLA [source URL consultée le 11 février 2021]. 
Vanja Manić-Matić

TEACHING FRENCH AS A FOREIGN LANGUAGE IN THE CONTEXT OF A PANDEMIC: TECHNOLOGICAL AND CULTURAL ASPECTS

\section{Summary}

Since the onset of the COVID-19 pandemic and the global health crisis, we are living in a new era that has imposed new habits and behaviors on us. The pandemic has been reflected in all areas of life and culture, including teaching/learning French as a foreign language. The interactions between humans have changed considerably, as well as the way of thinking and reacting. On the one hand, in teaching/learning French as a foreign language, the methodology and approach had to be adapted and have therefore become virtual. On the other hand, cultural exchanges have found a new way to manifest and develop. This in turn had a great impact on new strategies and on their structuring not only of thinking, but also of the methodology of FLE teaching/learning. The classroom management has been replaced by online management. Likewise, during the pandemic, new circumstances changed the culture and imposed new behaviors. Based on our own experience and on descriptive methodology, this work has several objectives: to present the technological and cultural aspects in force, to draw attention to the important changes linked to the pandemic and finally, to develop awareness of these new challenges with the focus of improving and deepening the teaching / learning of French as a foreign language.

Key words: French as a foreign language (FLE), teaching/learning, pandemic, technology, culture.

\section{RÉFÉRENCES BIBLIOGRAPHIQUES}

Byram, M. (1992). Culture et éducation en langue étrangère. Paris : Les Éditions Didier.

Bruneau, R. (26 mars 2020). Quarantaine [vidéo]. Disponible sur : https://www.youtube.com/watch?v=ccAkxNlumLA

CECR : Conseil de l'Europe. (2001). Cadre européen commun de référence pour les langues : apprendre, enseigner, évaluer (CECR). Strasbourg : Unité de Politiques Linguistiques. Consulté le 11 février 2021, disponible sur https://rm.coe.int/16802fc3a8

Chaves, R.-M.-Favier, L. \& Pélissier, S. (2012). L'Interculturel en classe. Grenoble : FLE PUG.

Cuq, J.-P.-Gruca, I. (2015). Cours de didactique du français langue étrangère et seconde. Grenoble : Presses universitaires de Grenoble. 
Defays, J.-M. (2018). Enseigner le français langue étrangère et seconde. Bruxelles : Éditions Mardaga.

Durbaba, 0. (2016). Kultura i nastava stranih jezika. Beograd: Univerzitet u Beogradu. Filološki fakultet.

Manić-Matić, V. (2017). Éléments de civilisation francophone dans l'enseignement $d u$ FLE. (Thèse de doctorat non publiée). Faculté de philosophie et lettres, Université de Novi Sad, Novi Sad

Manić-Matić, V. (2020). Le (non) verbal dans l'enseignement du FLE - enjeux, défis et réalités en contexte universitaire. Godišnjak Filozofskog fakulteta u Novom Sadu, XLV-4, 261-271.

Zarate, G. (1986). Enseigner une culture étrangère. Paris : Hachette

Zarate, G. (2016). Représentations de l'étranger et didactique des langues. Paris : Les Éditions Didier.

Polovina, P. (1980). Metodika nastave francuskog jezika. Beograd: Naučna knjiga.

Porcher, L. (1995). Le français langue étrangère. Paris: Hachette Éducation France.

Porcher, L. (2016). L'Enseignement des langues étrangères ». Paris : Hachette Éducation France.

Shiffler, L. (2006). Pour un enseignement interactif des langues étrangères.. Paris : Didier - Crédif, Collection LAL - Langues et Apprentissage des langues. 